\title{
Quantum Smoluchowski equation: Escape from a metastable state
}

\author{
Dhruba Banerjee ${ }^{\mathrm{a}}$, Bidhan Chandra Bag ${ }^{\mathrm{b}}$, \\ Suman Kumar Banik ${ }^{a}$ and Deb Shankar Ray ${ }^{a, *}$ \\ ${ }^{a}$ Indian Association for the Cultivation of Science, Jadavpur, Calcutta 700 032, \\ India \\ ${ }^{\mathrm{b}}$ Department of Chemistry, Visva-Bharati, Shantiniketan 731 235, India
}

\begin{abstract}
We develop a quantum Smoluchowski equation in terms of a true probability distribution function to describe quantum Brownian motion in configuration space in large friction limit at arbitrary temperature and derive the rate of barrier crossing and tunneling within an unified scheme. The present treatment is independent of path integral formalism and is based on canonical quantization procedure.
\end{abstract}

Key words: Quantum Smoluchowski equation, activated rate processes, tunneling PACS: 05.40.-a, 82.20.-w

\section{Introduction}

The theory of quantum Brownian motion is one of the major issues in physics and chemistry today. Its tremendous success in the treatment of various phenomena in quantum optics [1], quantum tunneling and coherence effects in condensed matter physics [2-4], activated processes in chemical physics [5-10] is now well-documented in the current literature. While the noise operator and density operator semigroup methods formed the core of development in quantum optics in sixties and seventies, real time path integrals attracted wide attention since eighties. However, these approaches are plagued with several difficulties. First, a search for quantum analogue of classical FokkerPlanck equation with a nonlinear potential, in general, leads us to equations

\footnotetext{
* Corresponding author, Tel.: +91 33 4733542, Fax: +91 334732805

Email address: pcdsr@mahendra.iacs.res.in (Deb Shankar Ray).
} 
of higher (than two) derivatives of quasi-probability distribution functions $[2,3,11]$. These distribution functions often become negative or singular in the full quantum regime. Second, although large coupling constants and large correlation times are treated nonperturbatively formally in an exact manner by functional integrals their analytic evaluation often poses severe difficulties as emphasized recently by Stockburger and Grabert [12] and one has to consider special cases and approximations, e.g., WKB or semiclassical limit. Moreover because of well-known sign problem the numerical evaluation of the path integrals are very difficult [12]. Third, in some situations [13] the theory does not retain its validity as the temperature $T \rightarrow 0$. This implies that vacuum fluctuations due to heat bath are not correctly taken into account. The question is how to extend classical theory to quantum domain for large friction at arbitrary temperature in terms of a true probabilistic description. Very recently we have addressed [10] this issue to develop a non-Markovian quantum Kramers' equation describing Brownian motion in a $c$-number phase space. The present analysis of overdamped limit is a brief offshoot of this development in configuration space. Our aim here is (i) to develop a quantum analogue of classical Smoluchowski equation valid for arbitrary temperature and (ii) to obtain the rate of escape from a metastable state due to thermal activation and tunneling within an unified description.

\section{The quantum Langevin equation}

To start with we consider the standard system-heat bath model whose Hamiltonian is given by

$$
\hat{H}=\frac{\hat{P}^{2}}{2}+V(\hat{X})+\sum_{j}\left[\frac{\hat{p}_{j}^{2}}{2}+\frac{1}{2} \kappa_{j}\left(\hat{q}_{j}-\hat{X}\right)^{2}\right]
$$

where $\hat{X}$ and $\hat{P}$ are the co-ordinate and momentum operators of the Brownian particle and the set $\left\{\hat{q}_{j}, \hat{p}_{j}\right\}$ is the set of co-ordinate and momentum operators for the reservoir oscillators coupled linearly to the system through the coupling constant $\kappa_{j}$ and obeying the usual commutation relation $[\hat{X}, \hat{P}]=i \hbar$ and $\left[\hat{q}_{j}, \hat{p}_{j}\right]=i \hbar \delta_{i j}$. The potential $V(\hat{X})$ is due to the external force field for the Brownian particle. Eliminating the bath degrees of freedom in the usual way [1] we obtain the operator Langevin equation for the particle

$$
\ddot{\hat{X}}(t)+\int_{0}^{t} d t^{\prime} \beta\left(t-t^{\prime}\right) \dot{\hat{X}}\left(t^{\prime}\right)+V^{\prime}(\hat{X})=\hat{F}(t)
$$


where the noise operator $\hat{F}(t)$ and the memory kernel $\beta(t)$ are given by

$$
\begin{aligned}
& \hat{F}(t)=\sum_{j}\left[\left\{\hat{q}_{j}(0)-\hat{X}(0)\right\} \kappa_{j} \cos \omega_{j} t+\hat{p}_{j}(0) \kappa_{j}^{1 / 2} \sin \omega_{j} t\right] \text { and } \\
& \beta\left(t-t^{\prime}\right)=\sum_{j} \kappa_{j} \cos \omega_{j}\left(t-t^{\prime}\right),
\end{aligned}
$$

respectively, with $\kappa_{j}=\omega_{j}^{2}$. Very recently [10] we have shown that on the basis of a quantum mechanical average $\langle\ldots\rangle$ over the bath modes with coherent states and the system with an arbitrary state Eq.(2) can be cast into the form of a generalized quantum Langevin equation in $c$-numbers,

$$
\ddot{x}+\int_{0}^{t} d t^{\prime} \beta\left(t-t^{\prime}\right) \dot{x}\left(t^{\prime}\right)+V^{\prime}(x)=f(t)+Q(x, t) .
$$

In writing Eq.(5) we denote the quantum mechanical mean value of position operator $\langle\hat{X}\rangle=x$ and $Q(x, t)=V^{\prime}(\langle\hat{X}\rangle)-\left\langle V^{\prime}(\hat{X})\right\rangle$. c-number quantum Langevin force $f(t)$ satisfies $\langle f(t)\rangle_{S}=0$ and

$$
\left\langle f(t) f\left(t^{\prime}\right)\right\rangle_{S}=\frac{1}{2} \sum_{j} \kappa_{j} \hbar \omega_{j}\left(\operatorname{coth} \frac{\hbar \omega_{j}}{2 k_{B} T}\right) \cos \omega_{j}\left(t-t^{\prime}\right) .
$$

Here $\langle\ldots\rangle_{S}$ corresponds to an ensemble average [10] over the quantum mechanical mean values of the co-ordinates and momenta of the bath oscillators. Eq.(6) is the celebrated quantum fluctuation-dissipation relation [2]. Quantum noise $f(t)$ and quantum fluctuation term $Q(x, t)$ are due to heat bath and nonlinearity of the potential, respectively. For details we refer to [10].

\section{Large friction limit and quantum Smoluchowski equation}

We now consider the diffusion of a particle in an external potential $V(x)$ as described by Eq.(5). In the overdamped limit we drop the inertial term $\ddot{x}$ and assume a Lorentzian density of modes of heat bath oscillators such that $\kappa(\omega) \rho(\omega)=(2 / \pi)\left[\gamma /\left(1+\omega^{2} \tau_{c}^{2}\right)\right]$, which in the limit $\tau_{c} \rightarrow 0$ results in a damping kernel $\beta\left(t-t^{\prime}\right)=\gamma \delta\left(t-t^{\prime}\right)$ in Eq.(5). $\rho(\omega)$ refers to the density function used in the continuum limit. Eq. (5) then assumes the form

$$
\dot{x}+\frac{1}{\gamma} V_{\text {quant }}^{\prime}(x, t)=\frac{f(t)}{\gamma}
$$


where we have expressed the effective quantum potential $V_{\text {quant }}(x, t)$ as $V_{\text {quant }}^{\prime}(x, t)$ $=V^{\prime}(x)-Q(x, t)$. Making use of the Stratonovich prescription the equivalent description of Eq.(7) in terms of true probability distribution $p(x, t)$ is given by

$$
\frac{\partial p(x, t)}{\partial t}=\frac{1}{\gamma} \frac{\partial}{\partial x}\left[V_{q u a n t}^{\prime} p(x, t)\right]+D_{q o} \frac{\partial^{2} p}{\partial x^{2}}
$$

Here $D_{q o}$ is the quantum diffusion coefficient which can be obtained with the following definition [1]

$$
2 D_{q o}=\frac{1}{\Delta t} \int_{t}^{t+\Delta t} d t_{1} \int_{t}^{t+\Delta t} d t_{2} \frac{1}{\gamma^{2}}\left\langle f\left(t_{1}\right) f\left(t_{2}\right)\right\rangle_{S}
$$

where the correlation function $\left\langle f\left(t_{1}\right) f\left(t_{2}\right)\right\rangle_{S}$ of the $c$-number quantum noise of the heat bath is given by Eq.(6). Making use of Eq.(6) in the continuum limit in Eq.(9) we obtain

$$
2 D_{q o}=\frac{1}{2 \gamma^{2} \Delta t} \int_{0}^{\infty} d \omega \kappa(\omega) \rho(\omega) \hbar \omega\left(\operatorname{coth} \frac{\hbar \omega}{2 k_{B} T}\right) I_{\omega}
$$

Here $I_{\omega}$ is given by $I_{\omega}=\int_{t}^{t+\Delta t} d t_{1} \int_{t}^{t+\Delta t} d t_{2} \cos \omega\left(t_{1}-t_{2}\right)$ which after explicit integration yields

$$
I_{\omega}=\frac{4}{\omega^{2}} \sin ^{2} \frac{\omega \Delta t}{2} .
$$

The general expression for quantum diffusion coefficient in the overdamped limit is therefore given by

$$
D_{q o}=\frac{2}{\gamma} \frac{1}{\pi \Delta t} \int_{0}^{\infty} \frac{1}{1+\omega^{2} \tau_{c}^{2}} \hbar \omega[2 \bar{n}(\omega)+1] \frac{1}{\omega^{2}} \sin ^{2} \frac{\omega \Delta t}{2} d \omega, \tau_{c} \rightarrow 0
$$

where we have put the Lorentzian density of states for bath oscillators as stated earlier. $\bar{n}(\omega)$ is the average thermal photon number of the heat bath and is given by $\bar{n}(\omega)=1 /\left[\exp \left(\hbar \omega / k_{B} T\right)-1\right]$. The quantum diffusion coefficient can thus be calculated exactly by numerical integration over the bath frequencies $\omega$ for $\tau_{c} \rightarrow 0$ (the Markovian limit). The following two limiting situations are further relevant for the present analytic treatment: (i) In the high temperature limit $k_{B} T \gg \hbar \omega$, the quantity $\hbar \omega[2 \bar{n}(\omega)+1]$ in Eq.(12) reduces to $k_{B} T$ and the explicit integration results in $D_{q o}=k_{B} T / \gamma$, the usual Einstein's value in the static friction limit. (ii) In the vacuum limit on the other hand $\bar{n}(\omega) \rightarrow 0$ 
and $D_{q o}$ may be obtained by considering the fact that the frequency dependence of the integrand in (12) [or (10)] except $I_{\omega}$ is flat (Markovian). This results in $D_{q o} \simeq \hbar \tilde{\omega} / 2 \gamma$, where $\tilde{\omega}$ is an average bath frequency which may be approximately taken to be the linearized frequency of oscillation in the well $\omega_{0}$ (i.e., $\tilde{\omega} \sim \omega_{0}$ ) since at $T \sim 0$ the dynamics is dominated by population at the bottom of the well. For an arbitrary intermediate temperature we must rely, however, on the general expression (12) for the quantum diffusion coefficient.

We now note that Eq.(8) contains quantum corrections to all orders. To make it more explicit we return to quantum mechanics of the system in the Heisenberg picture to express the operators $\hat{X}$ and $\hat{P}$ as

$$
\hat{X}(t)=\langle\hat{X}(t)\rangle+\delta \hat{X} \text { and } \hat{P}(t)=\langle\hat{P}(t)\rangle+\delta \hat{P}
$$

$\langle\hat{X}(t)\rangle$ and $\langle\hat{P}(t)\rangle$ are the quantities signifying quantum averages and $\delta \hat{X}$ and $\delta \hat{P}$ are quantum corrections. By construction $\langle\delta \hat{X}\rangle$ and $\langle\delta \hat{P}\rangle$ are zero and they obey commutation relation $[\delta \hat{X}, \delta \hat{P}]=i \hbar$. Using (13) in $V^{\prime}(\hat{X})$ and a Taylor expansion around $\langle\hat{X}\rangle(\equiv x)$ it is possible to express $Q(x, t)$ as $Q(x, t)=-\sum_{n \geq 2}(1 / n !) V_{n+1}(x)\left\langle\delta \hat{X}^{n}(t)\right\rangle$ and $V_{\text {quant }}(x, t)$ as

$$
V_{\text {quant }}(x, t)=V(x)+\sum_{n \geq 2} \frac{1}{n !} V_{n}(x)\left\langle\delta \hat{X}^{n}(t)\right\rangle
$$

where $V_{n}(x)$ is the $n$-th derivative of the the classical potential which gets modified by the quantum corrections. To solve quantum Smoluchowski equation (8) it is therefore necessary to calculate the corrections $\left\langle\delta \hat{X}^{2}(t)\right\rangle,\left\langle\delta \hat{X}^{3}(t)\right\rangle$, etc. To this end we return to operator Eq.(2) and make use of the relation (13) and a Taylor expansion of the potential $V(\hat{X})$ to derive the following coupled equations in the overdamped limit:

$$
\begin{aligned}
& \gamma \dot{x}+V^{\prime}(x)+\sum_{n \geq 2} \frac{1}{n !} V_{n+1}(x)\left\langle\delta \hat{X}^{n}(t)\right\rangle=f(t) \\
& \left\langle\delta \dot{X}^{n}(t)\right\rangle=-\frac{n}{\gamma} V^{\prime \prime}(x)\left\langle\delta \hat{X}^{n}(t)\right\rangle
\end{aligned}
$$

where $n=2,3, \ldots$. A decisive advantage in the treatment of overdamped limit is noteworthy. The equations for quantum corrections $\left\langle\delta \hat{X}^{n}(t)\right\rangle$ are closed in contrast to those for the system without its surrounding $[14,15]$. If one is interested in the local dynamics around a point $x_{0}$ (say, at the bottom or top of a potential well) the set of Eqs.(16) gets decoupled from (15) and one can obtain simple estimates of $\left\langle\delta \hat{X}^{n}(t)\right\rangle$ since $V^{\prime \prime}\left(x_{0}\right)$ can be treated as a constant in such cases. More generally however, Eq.(8) can be combined with Eq.(16) 
to provide an extended phase space description in terms of a true probability function $p\left(x, \eta_{2}, \eta_{3}, \ldots, t\right)$

$$
\begin{aligned}
\frac{\partial p\left(x, \eta_{2}, \eta_{3}, \ldots, t\right)}{\partial t}= & \frac{1}{\gamma} \sum_{n \geq 2} n \frac{\partial}{\partial \eta_{n}}\left[V^{\prime \prime}(x) \eta_{n} p\right]+\frac{1}{\gamma} \frac{\partial}{\partial x}\left[V^{\prime}(x) p\right] \\
& +\frac{1}{\gamma} \frac{\partial}{\partial x}\left[\sum_{n \geq 2} \frac{1}{n !} V_{n+1}(x) \eta_{n} p\right]+D_{q o} \frac{\partial^{2} p}{\partial x^{2}}
\end{aligned}
$$

where $\eta_{n}\left(\equiv\left\langle\delta \hat{X}^{n}(t)\right\rangle\right)$ for $n=2,3, \ldots$ span the space of variables signifying quantum corrections around quantum mechanical mean position $x$.

We now discuss the classical and vacuum limits of quantum Smoluchowski equation (8). As mentioned earlier in the classical limit, $D_{q o}$ reduces to Einstein's classical diffusion coefficient $k_{B} T / \gamma$. At the same time $Q(x, t)$ vanishes so that $V_{\text {quant }}(x, t)$ goes over to $V(x)$ and one recovers the usual classical Smoluchowski equation. In the opposite limit as $T \rightarrow 0$, however, both quantum noise due to system and vacuum fluctuation originating from the heat bath make significant contribution. $D_{q o}$ in this limit assumes the form $\hbar \omega_{0} / 2 \gamma$. In this context we refer to a recent treatment [13] of large friction limit in quantum dissipative dynamics to point out that the latter theory does not retain its full validity as $T \rightarrow 0$ since the quantum noise due to heat bath disappears in the vacuum limit. Another noteworthy feature of quantum Smoluchowski equation (8) [ or (17) ] is that unlike Wigner function based equations [11] it does not contain higher (than two) order derivatives of probability distribution function. The positive definiteness of this function is therefore ensured.

\section{Decay of a metastable state}

Based on the quantum Smoluchowski equation (8) we now consider the problem of escape from a metastable state. Over the last two decades this has attracted a lot of attention in classical theory of thermally activated processes [5]. Since a quantum analysis naturally includes tunneling as an integral part of the problem we turn to this issue in search of an unified description of tunneling and thermal activation.

To begin with we first take into consideration of the time-scale of free motion of the system, $\omega\left(\omega^{2}=V^{\prime \prime}\right)$. Since according to Eq.(16) quantum correction $\left\langle\delta \hat{X}^{n}(t)\right\rangle$ varies on a time-scale $\left(n \omega^{2} / \gamma\right)^{-1}$ it is convenient to make an average of this fluctuation over a period and replace $\left\langle\delta \hat{X}^{n}(t)\right\rangle$ by an average $\overline{\left\langle\delta \hat{X}^{n}\right\rangle}=$ $\omega \int_{0}^{1 / \omega}\left\langle\delta \hat{X}^{n}(t)\right\rangle d t$. This implies quantum noise does not change significantly on 
a time-scale during which the system relaxes so that $\tilde{V}(x)$ does not depend on time explicitly. Eq. (8) can then be recast in the form of continuity equation $\partial p(x, t) / \partial t=-\partial J / \partial x$ where $J$ can be identified as a current which is given by

$$
-J=\frac{1}{\gamma} \tilde{V}^{\prime}(x) p+\frac{D}{\gamma} \frac{\partial p}{\partial x}
$$

Here we have put

$$
\tilde{V}(x)=V(x)+\sum_{n \geq 2} \frac{1}{n !} V_{n}(x) \overline{\left\langle\delta \hat{X}^{n}\right\rangle}
$$

and $D=\gamma D_{q o}$. We now consider a cubic potential of the form $V(x)=$ $-(A / 3) x^{3}+B x^{2}$ ( $A$ and $B$ are positive constants), with a metastable minimum at $x=0$ which is separated from the true minimum by a finite potential barrier at $x=x_{b}$. Linearizing the potential $V(x)$ at the barrier top we calculate the stationary flux $J(\partial p / \partial t=0)$ around this point in the usual way,

$$
J=\frac{\omega_{b} \sqrt{D}}{\sqrt{2 \pi} \gamma} p(0) \exp (-E / D) \exp \left[\frac{-(1 / 2) V^{\prime \prime \prime}\left(x_{b}\right) \overline{\left\langle\delta \hat{X}^{2}\right\rangle_{b}} x_{b}}{D}\right]
$$

where $\omega_{b}$ refers to the frequency at the barrier top and $E$ is the activation energy $\left(E=V\left(x_{b}\right)\right)$. Again considering an equilibrium distribution or zero current situation $(J=0)$ around $x=0$ we linearize the potential $V(x)$ and derive from Eq.(18), the population in the well as given by

$$
n_{a}=\frac{\sqrt{2 \pi D}}{\omega_{0}} p(0)
$$

where $\omega_{0}$ refers to the frequency in the well at $x=0$. Based on the reasoning given earlier, we can obtain an estimate of quantum correction in (20) as ${\overline{\left\langle\delta \hat{X}^{2}\right\rangle_{x=x_{b}}}}=\left(\hbar / 2 \omega_{b}\right)\left[1+\left(2 \omega_{b} / \gamma\right)\right]$ which follows from the solution of Eq.(16) and a quantum average with minimum uncertainty state $\left\langle\delta \hat{X}^{2}(0)\right\rangle_{x=x_{b}}=$ $\left(\hbar / 2 \omega_{b}\right)$. Furthermore $x_{b}$ may be expressed as $x_{b}=\sqrt{2 E} / \omega_{0}$. With these simplifications the rate, $k$ is given by the ratio $J / n_{a}$ :

$$
k=\frac{\omega_{0} \omega_{b}}{2 \pi \gamma} \exp (-E / D) \exp \left[\frac{\left(\sqrt{2 E} / \omega_{0}\right)\left(\hbar A / 2 \omega_{b}\right)\left(1+\frac{2 \omega_{b}}{\gamma}\right)}{D}\right] .
$$

The expression (22) is the quantum rate of decay of a metastable state at any temperature. The quantum feature appears in two different ways. First, since 
$D$ may be treated as a quantum analogue of $k_{B} T$ in the Boltzmann exponential factor, which reduces to $\hbar \omega_{0} / 2$ in the vacuum limit and to $k_{B} T$ in the thermal limit, the quantum nature of the heat bath is manifested through $D$. Second, the quantum nature of the system is pronounced through quantum correction terms which are entangled with the nonlinearity of the potential and vanishes in the classical limit. Thus in the classical limit (22) reduces to the familiar expression for the Kramers'-Smoluchowski rate of escape [16],

$$
k_{c l}=\frac{\omega_{0} \omega_{b}}{2 \pi \gamma} \exp \left(-E / k_{B} T\right) .
$$

In the vacuum limit, i.e., at $T \sim 0$, on the other hand we have

$$
k_{v a c}=\frac{\omega_{0} \omega_{b}}{2 \pi \gamma} \exp \left(-2 E / \hbar \omega_{0}\right) \exp \left[\frac{A\left(\sqrt{2 E} / \omega_{0}\right)\left(\hbar / 2 \omega_{b}\right)\left(1+\frac{2 \omega_{b}}{\gamma}\right)}{(1 / 2) \hbar \omega_{0}}\right] \text {. }
$$

The vacuum contribution corresponds to quantum tunneling at zero temperature. It is important to recall that the problem of zero temperature tunneling was first successfully addressed by Caldeira and Leggett $[2,3]$ in early eighties which subsequently initiated a major advancement in the field of macroscopic quantum tunneling [5]. The main results in the context of overdamped limit can be summarized as follows: First, the damping causes an exponentially strong suppression of tunneling rate. Second, for strong dissipation there is a large region where thermal and quantum fluctuations interplay so that the total rate of decay increases because the thermal activation is supplemented by quantum tunneling at finite temperature. These features can be easily recovered in the present theory. The rate coefficient in the vacuum limit $k_{v a c}$ shows that as the temperature approaches zero tunneling decay is almost exponential. Furthermore, at finite temperature, the presence of factor $(\hbar A) /\left(D \omega_{b}\right)$ in the exponential in the total rate coefficient in Eq.(22) implies an interplay of thermal fluctuation in $D$ and quantum fluctuation due to $(\hbar A) / \omega_{b}$ arising out of nonlinearity of the system. This leads to a effective reduction of the activation barrier from its classical value $E$ resulting in an increase of the rate of decay. It is thus apparent that although the results are qualitatively comparable the underlying physical picture in the two schemes (the path integral and the present one) are different. The tunneling decay at low temperature in the formal case is primarily determined by the nature of bounce solution evaluated by a semiclassical steepest decent method which depends on the effective path between the turning points. At the high temperature (i.e., in the classical regime) an unified multidimensional WKB approach is generally advocated to recover classical result $[4,5]$. The classical theory in the present formulation on the other hand is contained in $k_{c l}$ (Eq.(23)) which shows that the rate of thermal activation varies inversely with damping constant according to Kramers'-Smoluchowski limit. The quantum rate coefficient (22) thus 
interpolates between high temperature thermal activation and zero temperature tunneling.

\section{Conclusions}

In conclusion, we have considered the strong friction limit in quantum stochastic processes to develop a quantum Smoluchowski equation. The rate of decay of a metastable state which includes thermal activation and tunneling within a single scheme has been derived. Since classical Smoluchowski equation has a diverse range of applicability, e.g., in fluctuating barrier [17], in thermal ratchet and molecular motors [18-20], in tunnel diodes [21] and in the decay of periodically driven metastable potentials [22-24] we hope the present quantum analogue to keep its potential in similar issues.

This work was supported by the Council of Scientific and Industrial Research (C.S.I.R.), Government of India, under grant No. 01/(1740)/02/EMR-II.

\section{References}

[1] W. H. Louisell, Quantum Statistical Properties of Radiation, Wiley (New York, 1973).

[2] A. O. Caldeira and A. J. Leggett, Phys. Rev. Lett. 46 (1981) 211.

[3] A. O. Caldeira and A. J. Leggett, Ann. Phys. (N.Y.) 149 (1983) 374.

[4] U. Weiss, Quantum Dissipative Systems, World Scientific (Singapore, 1993).

[5] P. Hänggi, P. Talkner and M. Borkovec, Rev. Mod. Phys. 62 (1990) 251.

[6] P. G. Wolynes, Phys. Rev. Lett 47 (1981) 968.

[7] W. H. Miller, H. D. Schwartz and J. W. Tromp, J. Chem. Phys. 79 (1983) 4889.

[8] J. Liao and E. Pollak, J. Chem. Phys. 110 (1999) 80.

[9] J. Ray Chaudhuri, B. C. Bag and D. S. Ray, J. Chem. Phys. 111 (1999) 10852.

[10] D. Banerjee, B. C. Bag, S. K. Banik and D. S. Ray, Phys. Rev. E 65 (2002) 021109.

[11] W. H. Zurek and J. P. Paz, Phys. Rev. Lett. 72 (1994) 2508.

[12] J. T. Stockburger and H. Grabert, Phys. Rev. Lett. (in press).

[13] J. Ankerhold, P. Pechukas and H. Grabert, Phys. Rev. Lett. 87 (2001) 086802. 
[14] A. K. Pattanayak and W. C. Schieve, Phys. Rev. E 50 (1994) 3601.

[15] B. Sundaram and P. W. Milonni, Phys. Rev. E 51 (1995) 1971.

[16] H. A. Kramers, Physica 7 (1940) 284.

[17] C. R. Doering and J. C. Gadoua, Phys. Rev. Lett. 69 (1992) 2318.

[18] M. O. Magnasco, Phys. Rev. Lett. 71 (1993) 1477.

[19] R. D. Astumian, Science 276 (1997) 917.

[20] F. Jülicher, A. Ajdari and J. Prost, Rev. Mod. Phys. 69 (1997) 1269.

[21] R. N. Mantegna and B. Spagnolo Phys. Rev. Lett. 84 (2000) 3025.

[22] V. N. Smelyanski, M. I. Dykman, B. Golding, Phys. Rev. Lett. 82 (1999) 3193.

[23] J. Lehmann, P. Reimann and P. Hänggi, Phys. Rev. Lett. 84 (2000) 1639.

[24] R. S. Maier and D. L. Stein, Phys. Rev. Lett. 86 (2001) 3942. 